\title{
On the relationship between magnetic viscosity and coercivity of perpendicular media
}

\author{
J. G. Th. te Lintelo and J. C. Lodder \\ University of Twente, P. O. Box 217, 7500 AE Enschede, The Netherlands
}

(Received 20 January 1994; accepted for publication 15 April 1994)

\begin{abstract}
The dependent and dc-demagnetization measurements were performed on two types of perpendicular thin film media, $\mathrm{Co}-\mathrm{Cr}$ and $\mathrm{Fe}$-alumite. From these the parameters for magnetic viscosity and irreversible susceptibility were derived. Their ratio, resulting in the fluctuation field, is shown to relate logarithmically to the coercivity. This so-called "Barbier plot for perpendicular media" is found to exhibit a slope of approximately one and a half instead of one, as is found for in-plane media. The activation volumes were also derived and related logarithmically to the coercivity. Here a slope of approximately minus one and a half was found, instead of minus 0.73 as is found for in-plane media.
\end{abstract}

\section{INTRODUCTION}

The phenomenon of magnetic viscosity in recording media is of practical interest in long term stability, high frequency writing, and print through. ${ }^{1}$ It describes the relaxation of a metastable systern towards a more stable state, and has its origin in a thermally activated transition over energy barriers. For a single energy barrier this relaxation is determined by the well-known Arrhenius-Néel law. When a dispersion in energy barriers is present, however, the magnetic viscosity $S$ is derived from

$$
M(t)=M(0)+S \ln (t),
$$

where $M$ is the magnetization at time $t$, or at the, start of the measurement $(t=0)$. Use of this equation is generally found to be applicable over 2-3 decades' and is therefore a valid and useful description of experimental data. ${ }^{2}$

Research on time dependent effects is mainly focused on in-plane particulate materials. This has resulted in associating $S$ with the microscopic structure of materials and processes responsible for magnetization reversal. ${ }^{2}$ However, for continuous thin film media like ME tape or perpendicular $\mathrm{Co}-\mathrm{Cr}$, this description is thought not to be adequate, due to the presence of a strong internal and time dependent demagnetizing field. ${ }^{3}$ Because in future recording media (i.e., continuous and/or highly packed particulate materials) the demagnetizing field will become increasingly important, we investigated the relationship between $S$ and the demagnetizing field in a previous paper. ${ }^{4}$ There it was found that for Co-Cr microstrips with typical dimensions of $1-5 \mu \mathrm{m}$ and with varying demagnetization factors $N_{z}$ the magnetic viscosity $S$ increased with decreasing $N_{z}$. The slope of the hysteresis curve, however, also increased with decreasing $N_{z}$, resulting in a linear relation between $S$ and the total susceptibility. This result compares well with the concept of a fluctuation field $H_{f}$ (Ref. 5) for in-plane media, i.e.,

$$
H_{f}=\frac{S}{\chi_{i}^{\mathrm{inT}}},
$$

where $\chi_{i}^{\mathrm{i} r \mathrm{r}}$ represents the irreversible component of the internal susceptibility. Note that in Ref. 4 the total external susceptibility was applied.
The fluctuation field can be related to volumes of material which are involved in the activation process and is therefore of great interest in explaining magnetic reversal behavior. The relation is given by ${ }^{6}$

$$
H_{f}=\frac{k T}{d E / d H}=\frac{k T}{\mu_{0} v M_{s}},
$$

where $E$ is the activation energy, $H$ the magnetic field, and $v$ the activation volume. The activation volumes can be related to coercivity and it was found experimentally that in-plane materials obey the relation ${ }^{6}$

$$
H_{c} \propto v^{-x} \text { where } x=0.73 \text {. }
$$

The fluctuation field can also be related to the coercivity. This is expressed experimentally in so-called Barbier plots, where both coercivity and fluctuation field are plotted logarithmically ${ }^{7}$. For in-plane media this results in a linear relationship:

$$
\log \left(H_{f}\right)=\log \left(H_{c}\right)+C,
$$

where $C$ is a constant. The theoretical description of Eq. (5) was derived by Liu et al. ${ }^{8}$ and reveals information on the activation energy for different reversal models.

Whereas the time dependent effects for perpendicular media are thought to be qualitatively different, ${ }^{3}$ in this paper the relationship between coercivity, activation volume and fluctuation field is examined for $\mathrm{Co}-\mathrm{Cr}$ and $\mathrm{Fe}$-alumite. This results in an extension of the Barbier plot for perpendicular media. As such, this paper contributes to the discussion on correlating activation volume, fluctuation field, and coercivity, as initiated by Wohlfarth. ${ }^{6}$

The present paper is organized as follows: in Sec. II the influence of demagnetization on the magnetic viscosity is reviewed; in Sec. III the experimental conditions are presented; in Sec. IV the results are discussed and in Sec. V a summary of the conclusions is given.

\section{MAGNETIC AFTEREFFECT IN PERPENDICULAR MEDIA}

The magnetic aftereffect in perpendicular media is complicated by the presence of a strong time dependent demag- 
TABLE I. Magnetic and structural properties of the Fe-alumite samples.

\begin{tabular}{cccccr}
\hline $\begin{array}{c}\text { Sample } \\
\text { name }\end{array}$ & $\begin{array}{c}\text { Pore diameter } \\
(\mathrm{nm})\end{array}$ & $\begin{array}{c}\text { Cell diameter } \\
(\mathrm{nm})\end{array}$ & $\begin{array}{c}M_{s} \\
(\mathrm{kA} / \mathrm{m})\end{array}$ & $\begin{array}{c}H_{x_{\text {perp }}} \\
(\mathrm{kA} / \mathrm{m})\end{array}$ & $\begin{array}{c}K_{u} \\
\left(\mathrm{~kJ} / \mathrm{m}^{3}\right.\end{array}$ \\
\hline $\mathrm{Fe1}$ & 17.5 & 40.5 & 271 & 186 & 70 \\
$\mathrm{Fe} 2$ & 22.5 & 40.5 & 353 & 173 & 47 \\
$\mathrm{~A} 11$ & 45.0 & 117 & 258 & 80 & 51 \\
$\mathrm{~A} 21$ & 55.0 & 117 & 369 & 50 & 30 \\
A32 & 72.0 & 117 & 616 & 32 & -59 \\
B11 & 30.0 & 74.5 & 240 & 147 & 54 \\
B33 & 45.0 & 74.5 & 525 & 73 & 17 \\
\hline
\end{tabular}

netizing field. As a result some distinct characteristics arise, i.e., the relaxation process is quasi-logarithmic and the coefficient of viscosity is nearly independent of the applied field over a range of several $100 \mathrm{kA} / \mathrm{m}$., ${ }^{4-11}$ This last observation can possibly be attributed to the relationship of $S$ with the susceptibility ${ }^{4}$ and the almost constant behavior of $H_{f}$ with field [Eq. (2)]. In order to investigate this relationship for perpendicular media and to relate it to the coercivity the internal irreversible susceptibility $\chi_{i}^{\mathrm{irr}}$ should be derived [see Eq. (2)].

The internal field can be rewritten as an expression where the demagnetization factor $N_{z}$ of the sample is taken into account. So Eq. (2) may be written as ${ }^{12}$

$$
H_{f}=\frac{S}{\chi_{a}^{\mathrm{irr}}}\left(1-N_{z} \chi_{a}^{\mathrm{rev}}\right),
$$

where $\chi_{a}^{\mathrm{irr}}$ denotes the irreversible susceptibility, measured as the change in intensity of magnetization per unit change of applied field, and $\chi_{a}^{\text {rey }}$ denoles the reversible component. Note that $S$ in Eq. (6) now represents the coefficient of magnetic viscosity measured from a sample with nonzero $N_{z}$.

For particulate and in-plane materials, which consist of noninteracting particles, the irreversible susceptibility is in general derived from dc-demagnetization-remanence curves (DCD). This seems to be inappropriate for perpendicular and continuous media, because the elimination of the reversibility can contain a small irreversible component. ${ }^{13}$ However, because at zero applied field this effect and the reversible component are expected to be "small, here the irreversible susceptibility is also calculated from DCD-remanence curves.

The fluctuation field is often regarded as a constant intrinsic material property which implies that it is shape independent. This conclusion was drawn by Liu et al. ${ }^{8}$ and experimentally indicated in Ref. 4 and 14. Chantrell et al., ${ }^{15}$ however, showed theoretically that this is not true for aligned Stoner-Wohlfarth particles and experimentally verified this for $\gamma-\mathrm{Fe}_{2} \mathrm{O}_{3}$ data tape.

A model for relaxation in perpendicular thin films has been derived by Chantrell et al. ${ }^{16}$ and was found to give excellent agreement with experimental data on Fe-alumite films. This relationship between the magnetic viscosity and the energy barrier of a Stoner-Wohlfarth particle is given by

$$
S_{r}=\sqrt{\frac{k T}{25 K_{u} V}}
$$

where $S_{r}$ denotes the magnetic viscosity coefficient at zero applied field normalized on the remanence. When the reversible component of the susceptibility is disregarded, i.e., $\chi_{\text {irr }}=\chi_{\text {tot }}=M_{r} / H_{c}$, this can be rewritten as

$$
H_{f}=H_{c} \sqrt{\frac{k T}{25 K_{u} V}}
$$

The right-hand side of Eq. (7) is related to the energy barrier of an incoherent magnetic reversal. How this is connected to the coercivity of perpendicular media is not known. But it is likely that Eq. (8) will result in a linear logarithmic relationship with a slope larger than one. Therefore, anomalous behavior is expected in comparison with in-plane media.

\section{EXPERIMENT}

In this study two types of perpendicular magnetized media are investigated, i.e., $\mathrm{Co}-\mathrm{Cr}$ as a continuous and $\mathrm{Fe}-$ alumite as a particulate medium. The $\mathrm{Co}-\mathrm{Cr}$ films are rf sputtered and varied in thickness, saturation magnetization, and magnetic anisotropy. The Fe-alumite films consist of small iron needles and are varied in diameter and packing densities. The most interesting parameters for this study are listed in Tables I and II. Here $K_{u}$ stands for the first order uniaxial anisotropy constant of the whole film and $K_{l}$ stands for the first-order crystal anisotropy constant. For a more extensive description of these films the reader is referred to Refs. 4,17 , and 18.

The magnetic viscosity and irreversible properties are measured with a vibrating sample magnetometer (VSM) with the field perpendicular to the film plane. For the time dependent measurements, use was made of a home-built VSM with a maximum applicable field of $1.2 \mathrm{~T}$, which is obtained from

TABLE II. Magnetic and structural properties of the Co-Cr samples.

\begin{tabular}{lccccc}
\hline \hline $\begin{array}{l}\text { Sample } \\
\text { name }\end{array}$ & $\begin{array}{c}\text { Cr contcnt } \\
\text { (at. \%Cr) }\end{array}$ & $\begin{array}{c}\text { Thickncss } \\
(\mathrm{nm})\end{array}$ & $\begin{array}{c}M_{s} \\
(\mathrm{kA} / \mathrm{m})\end{array}$ & $\begin{array}{c}H_{c \text { per. }} \\
(\mathrm{kA} / \mathrm{m})\end{array}$ & $\begin{array}{r}K_{l}^{+} \\
\left(\mathrm{kJ} / \mathrm{m}^{3}\right)\end{array}$ \\
\hline Rf46 & 19 & 46 & 442 & 88 & 94 \\
Rf110 & 19 & 110 & 467 & 101 & 115 \\
Rf297 & 19 & 297 & 444 & 65 & 99 \\
Rf611 & 20 & 611 & 425 & 49 & 84 \\
Rf982 & 19 & 982 & 427 & 43 & 87 \\
$120291-10$ & 23 & 560 & 293 & 66 & 69 \\
$140391-8$ & 21 & 485 & 470 & 74 & 140 \\
$140391-10$ & 21 & 490 & 467 & 83 & 140 \\
\hline
\end{tabular}



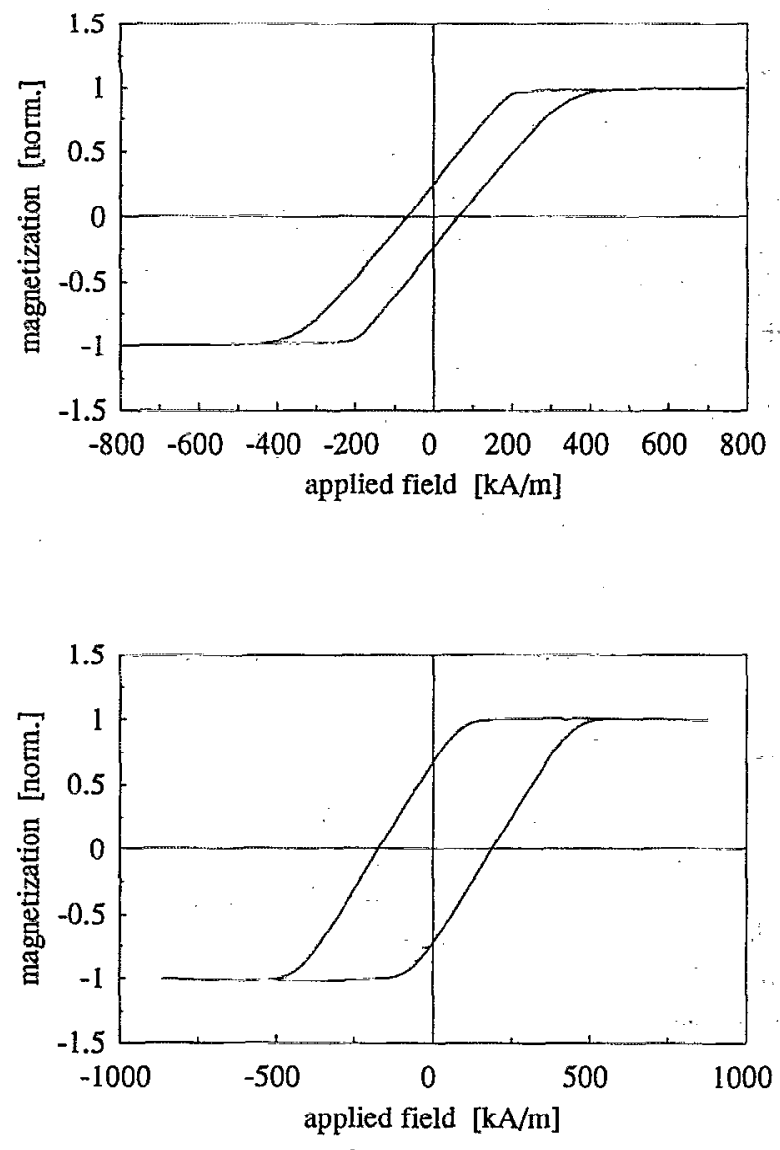

FIG. 1. Typical example of a perpendicular hysteresis curve of the perpendicular media $\mathrm{Co}-\mathrm{Cr}$ (top, sample 120291-10) and Fe-alumite (bottom, sample Fe 1).

an electromagnet. The DCD measurements were obtained from a commercially available Oxford Instruments VSM, which is equipped with a $3 \mathrm{~T}$ superconducting magnet. All measurements were performed at room temperature.

For time dependent measurements the films were first saturated to $-1000 \mathrm{kA} / \mathrm{m}$ before setting the field to zero field. The magnetic moment was then monitored for $1 \mathrm{~h}$. The magnetic viscosity was obtained by fitting the normalized magnetization to Eq. (1). The starting time $(t=0)$ is chosen to be $6 \mathrm{~s}$ after the sudden change in the applied field, in order to circumvent time dependent effects of the VSM. The error in $S$ is assumed to be around 15\%. For an example of the magnetic after-effect measurement of these films the reader is referred to Ref. 4.

The irreversible susceptibility is derived by differentiating the DCD-demagnetization curves.

\section{RESULTS AND DISCUSSION}

In this section the relations between susceptibility, magnetic viscosity, and coercivity are discussed for perpendicular media. Perpendicular media exhibit a strong demagnetizing field which influences magnetic behavior and results in typical tilted hysteresis curves. In Fig. 1 an example is shown of the perpendicular hysteresis curves of a $\mathrm{Co}-\mathrm{Cr}$ and $\mathrm{Fe}$ alumite sample, respectively.

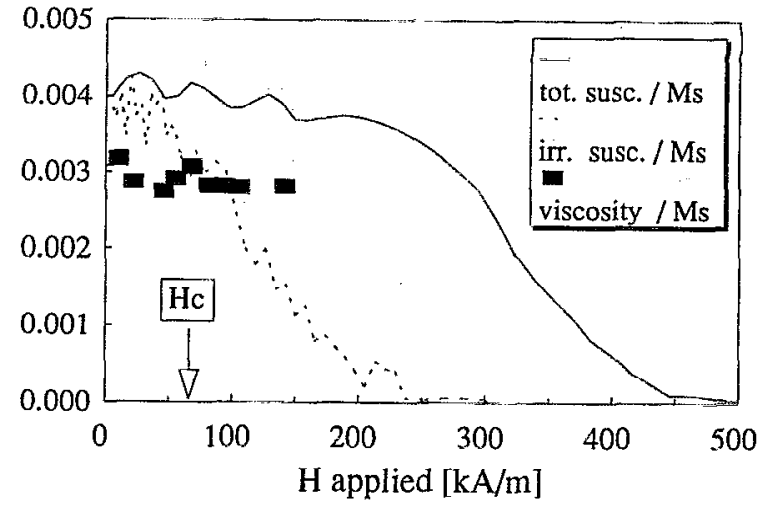

FIG. 2. Magnetic susceptibility (total and irreversible component) and magnetic viscosity as a function of the applied field for $\mathrm{Co}-\mathrm{Cr}$ sample 120291-10.

From Fig. 1 it can be derived that the total magnetic susceptibility will show an almost constant behavior. This behavior is illustrated in Fig. 2 for a Co-Cr sample, where both the irreversible and total susceptibility are given as a function of the applied field.

The irreversible component almost equals the total susceptibility around zero, but then falls off more rapidly with the field. This behavior is attributed to the self-reorientation effect ${ }^{13}$ and makes application of DCD-curves for obtaining the irreversible susceptibility at higher fields questionable. The reversible component of the susceptibility around the zero applied field, i.e., the field of interest here, is, however, small. This conclusion applies to all films investigated here.

In Fig. 2 the magnetic viscosity $S$ is also plotted as a function of the applied field. Note that a broad field dependence is present and thus there is no maximum value of $S$ near coercivity as is observed for in-plane media. ${ }^{1}$ This observation is in agreement with Refs. 9 and 11.

In Fig. 3 the relationship between $H_{f}$ and the coercivity is plotted logarithmically and represents the so-called Barbier plot for perpendicular media. The samples can be fitted to a line with approximate slope 1.5 and therefore suggest disagreement with Eq. (5). Note that the line connecting the alumite samples is lower than the line connecting the $\mathrm{Co}-\mathrm{Cr}$

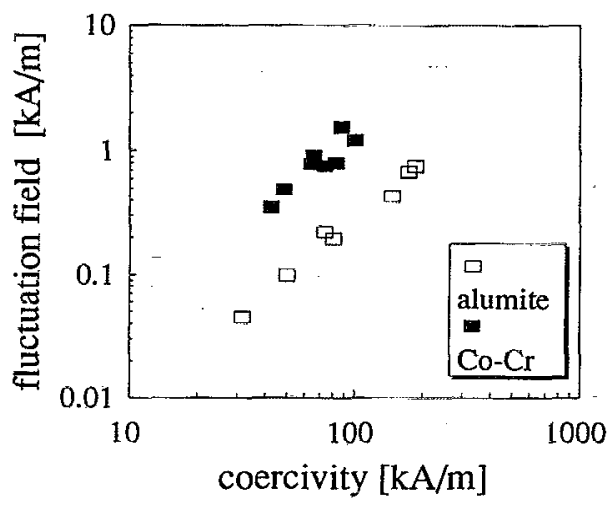

FIG. 3. The fiuctuation field as a function of coercivity. 


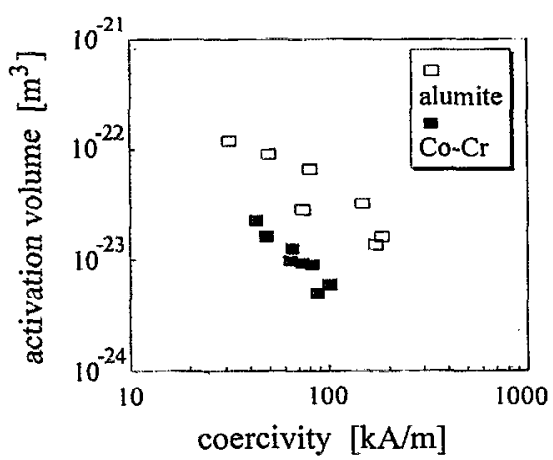

FIG. 4. The activation volume as a function of coercivity.

samples. This may possibly be attributed to a different reversal mechanism, resulting in smaller effective activation volumes for the $\mathrm{Co}-\mathrm{Cr}$ samples.

In Fig. 4 this is investigated by logarithmically plotting the activation volumes against the coercivity. From Fig. 4 it can be seen that the Co-Cr films exhibit smaller activation volumes than the $\mathrm{Fe}$-alumite samples, and that both curves can be fitted to a line with a slope of $\sim-1.5$. This disagrees with Eq. (4) and therefore suggests a strong influence of the demagnetizing field on the reversal mechanism.

The activation volumes are in the region of one to two orders of magnitude above the superparamagnetic limit. For the Co-Cr samples its value agrees with switching volumes as found for similar samples in the literature. ${ }^{19}$ For the Fealumite samples its value is around 20 times as small as its particle volume. ${ }^{16}$ In Fig. 5 this behavior is denoted, from which an incoherent reversal mode is concluded.

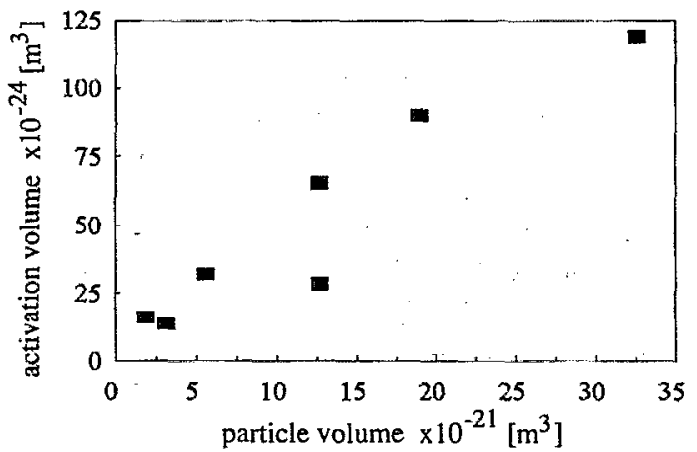

FIG. 5. The activation volumes of Fe-alumite as a function of its particle volume.

\section{CONCLUSIONS}

In this article time dependent effects of two types of perpendicular thin film media, $\mathrm{Co}-\mathrm{Cr}$ and $\mathrm{Fe}$-alumite, are investigated. From this it can be concluded that the fluctuation field relates logarithmically to the coercivity. A linear relationship is obtained with a slope of $\sim 1.5$ instead of 1 , as for in-plane media. The activation volume is also found to relate logarithmically to the coercivity. Here a linear relationship is obtained with a slope of $\sim-1.5$ instead of -0.73 , as for in-plane media.

This anomalous behavior of perpendicular films, in comparison with in-plane media, is attributed to the presence of a strong demagnetizing field. This field is thought to influence the reversal mechanism considerably. Time dependent measurements on perpendicular films are therefore considered of interest in explaining micromagnetic behavior.

\section{ACKNOWLEDGMENTS}

This research was carried out within the frame work of the EC Community Action on Magnetic Storage Technology (CAMST). The authors would like to thank Eelco Sterringa for measuring the alumite samples and $Y$. Wakui of the Yamaha Research and Development Center for providing the alumite samples.

${ }^{1}$ M. P. Sharrock, IEEE Trans. Magn. MAG-26, 193 (1990).

${ }^{2}$ R. W. Chantrell, Nato ASI Series E, edited by K. H. J. Buschow, G. J. Long, and F. Grandjean, Vol. 229, Chap. 4 (1993).

${ }^{3}$ A. Lyberatos, R. W. Chantrell, E. R. Sterringa, and J. C. Lodder, J. Appl. Phys. 70, 4431 (1991).

${ }^{4}$ J. G. Th. te Lintelo, W. Streekstra, J. C. Lodder, and Th. J. A. Popma, IEEE Trans. Magn. MAG-29, 3748 (1993).

${ }^{5}$ R. Street and J. C. Woolley, Proc. R. Phys. Soe. A 62, 562 (1949).

${ }^{6}$ E. P. Wohlfarth, J. Phys. F 14, L155 (1984).

${ }^{7}$ J. C. Barbier, Annal. Phys. 9, 84 (1954).

${ }^{8}$ J. F. Liu and H. L. Luo, J. Magn. Magn. Mater. 86, 153 (1990).

${ }^{9}$ D. K. Lottis, E. D. Dahlberg, J. A. Christner, J. I. Lee, R. L. Peterson, and R. M. White, J. Phys. (Paris) Colloq. 49, Suppl., C8-1989 (1988).

${ }^{10}$ D. Lottis, R. White, and E. D. Dahlberg, J. Appl. Phys. 67, 5187 (1990).

"1 B. C. Webb, S. Schultz, and S. B. Oseroff, J. Appl. Phys. 63, 2923 (1990)

${ }^{12}$ R. Street, R. K. Day, and J. B. Dunlop, J. Magn. Magn. Mater. 69, 106 (1987).

${ }^{13}$ T. Maro and O. Kitakami, Jpn. J. Appl. Phys. 32, 3448 (1993).

${ }^{14}$ S. Ke, L. J. Fang, and L. H. Lie, J. Phys. D 23, 439 (1990).

${ }^{15}$ R. W. Chantrell, J. Magn. Magn. Mater. 95, 365 (1991).

${ }^{16}$ R. W. Chantrell, A. I yheratos, E. R. Sterringa, and J. C. Lodder, Abstract Book ICMFS P1.6, P41 (1991) (unpublished).

${ }^{17}$ W. J. M. A. Geerts, J. G. Th. te Lintelo, J. C. Lodder, and Th. J. A. Popma, IEEE Trans. Magn. 26, 36 (1990).

${ }^{18}$ J. C. Lodder and L. C. Zhang, IEEE Trans. Magn. 25, 4171 (1989).

${ }^{19} \mathrm{~S}$. de Haan and J. C. Lodder (unpublished). 